\title{
Suspended Microracetrack Resonator with Lateral Sub-wavelength-Grating Metamaterial Cladding for Mid-infrared Sensing Applications
}

\author{
Zecen Zhang ${ }^{1}$, Geok Ing $\mathrm{Ng}^{1}$, Ting $\mathrm{Hu}^{1}$, Haodong $\mathrm{Qiu}^{1}$, Xin Guo ${ }^{1}$, Wanjun Wang ${ }^{1}$, \\ Mohamed S. Rouifed ${ }^{1}$, Chongyang Liu ${ }^{1}$, Jiaxu $\mathrm{Sia}^{1}$, Jin Zhou ${ }^{1}$, and Hong Wang ${ }^{1}$ \\ ${ }^{1}$ NOVITAS, Nanoelectronics Centre of Excellence, School of Electrical and Electronic Engineering, \\ Nanyang Technological University, 639798 Singapore
}

\begin{abstract}
A one-time etching suspended microracetrack resonator with lateral sub-wavelength-grating (SWG) metamaterial cladding is theoretically and experimentally demonstrated on commercial $340 \mathrm{~nm}$ thick-top-silicon silicon-on-insulator (SOI) platform for mid-infrared (MIR) bio-chemical sensing applications. The suspended structure can offer a larger exposed area of waveguides with the testing chemicals as well as a decent sensitivity. And the one-time etching process also eases the fabrication. The suspended waveguide is optimized with a balance between propagation loss and the sensitivity. The suspended microracetrack resonator is experimentally measured at $2 \mu \mathrm{m}$ wavelength and well fitted with an extinction ratio (ER) of $12.3 \mathrm{~dB}$ and a full-width-at-half-maximum (FWHM) of $0.12 \mathrm{~nm}$, which corresponds to a quality factor (Q factor) of 16600. With the equivalent refractive index method and a specially developed numerical model, the expected sensitivities of fundamental TE and TM mode were calculated as $58 \mathrm{~nm} / \mathrm{RIU}$ and $303 \mathrm{~nm} / \mathrm{RIU}$ respectively. This one-time etching suspended microracetrack resonator shows great potential in MIR optical bio-chemical sensing applications.
\end{abstract}

\section{Introduction}

Recently, silicon photonics based on silicon-on-insulator (SOI) platform has attracted a lot of research interest, since it can utilize the industrial mature fabrication process with lower cost. Many efforts have been invested on the SOI based bio-chemical sensing in the nearinfrared (NIR) band, typically around 1.3-1.6 $\mu \mathrm{m}$ wavelength [1-6]. Various structures and methods have been utilized to achieve high sensitivity [7-18]. However, limited work has been reported on mid-infrared (MIR) sensors based on the SOI platform. One of the limitation of MIR sensing based on SOI platform is the large absorption in $\mathrm{SiO}_{2}$ at the wavelengths longer than $4 \mu \mathrm{m}$. One solution is the suspended structures with the buriedtesting material hence increase the sensitivity [19-22]. In 2012, a suspended membrane waveguide based microring resonator working at $2.75 \mu \mathrm{m}$ was reported by $\mathrm{Z}$. Cheng et al.

Corresponding author: ZZHANG023@e.ntu.edu.sg 
[23]. In 2013, Y. Xia et al. experimentally reported a suspended Si resonator working at 3.4 $\mu \mathrm{m}$ and $5.2 \mu \mathrm{m}[24]$. But for these two structures, multiple etching processes are needed, which include a partial etching of the rib waveguide and a full etching of the via holes for the following hydrofluoric acid (HF) etching. Furthermore, the wide and thin slabs beside the rib waveguide, which target to suppress the mode leakage, limit the mechanical stability of the system.

In this manuscript, for the first time, we theoretically and experimentally demonstrate a one-time etching suspended microracetrack resonator with lateral sub-wavelength-grating (SWG) metamaterial cladding on a commercial SOI platform for MIR bio-chemical sensing application. A quality factor (Q factor) of 16000 and an extinction ratio (ER) of $13.4 \mathrm{~dB}$ were measured at $2 \mu \mathrm{m}$ wavelength. Furthermore, with the equivalent refractive index method and a specially developed numerical model, the expected sensitivities of fundamental TE and TM mode were calculated as $58 \mathrm{~nm} / \mathrm{RIU}$ and $303 \mathrm{~nm} / \mathrm{RIU}$. This system shows great potential for the MIR band sensing applications.

\section{Device designs, fabrication and characterization}

The one-time etching suspended microracetrack resonator with lateral SWG metamaterial cladding are designed on a commercial SOI wafer with $340 \mathrm{~nm}$-thick-top-silicon and $2 \mu \mathrm{m}$ thick buried $\mathrm{SiO} 2$ (BOX) layer. The cladding layer is air. At first, designing and optimizing the fundamental building block namely the suspended waveguide were carried out. Fig. 1 shows the schematic of the suspended waveguide.

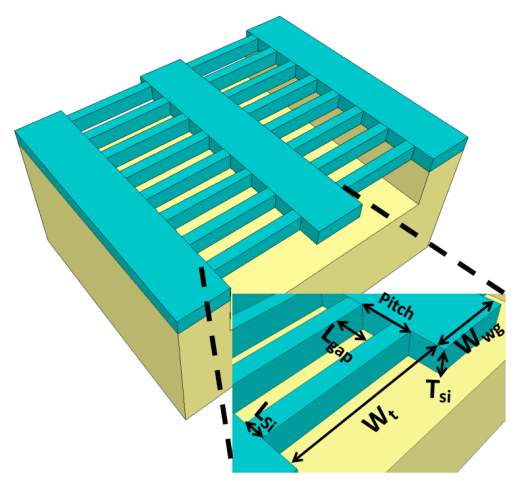

Fig. 1. The schematic of the one-time etching suspended waveguide with lateral SWG metamaterial cladding

For the optimization of the suspended waveguide, three crucial points need to be addressed: 1. Diffractive effects and back-reflections; 2. Mechanical stability; 3. Enhance the overlap between the evanescent field and the testing chemicals. First, in order to suppress the diffractive effect and back-reflections, the pitch of the sub-wavelength grating ( Pitch $=L_{s i}+L_{\text {gap }}$ ) needs to follow the Bragg condition [21]:

$$
\text { Pitch }<\text { Pitch }_{B g}=\frac{\lambda}{2 n_{B-F}}
$$

where Pitch $_{B g}$ is the Bragg period; $\lambda$ is the working wavelength; $n_{B-F}$ is the effective index of the fundamental Bloch-Floquet mode propagating in the waveguide. Second, in order to obtain a robust structure to overcome the fluctuations caused by testing liquid or gas flows, we need to have wide silicon pillars. However, it is known that the sensitivity is determined by the overlap between the evanescent field and the testing chemicals [25]. The 
trade-off is that while the duty-cycle $\left(D C=L_{s i} /\left(L_{s i}+L_{g a p}\right)=L_{s i} /\right.$ Pitch $)$ increases with the pitch fixed, the exposed area of waveguide becomes smaller as well as the overlap between the evanescent field and the testing chemicals, which results in a degradation of sensitivity. Hence, a balance between the mechanical stability and the sensitivity needs be considered under the precondition of a small enough Pitch that can suppress diffractive effect and back-reflections. The width of the lattice is set as $2 \mu \mathrm{m}$ to prevent the lateral energy leakage. Another two important influencing factors are the width and the thickness of the waveguide. The reduction of the waveguide cross-section area will compress the mode field. Consequently, more energy will gather at the surface of the waveguide thus bringing a higher overlap between the evanescent field and the testing chemicals but at the expense of a higher propagation loss. This is another trade-off which needs to be taken into account.

In order to test and verify the above discussions, one-time suspended waveguides with different dimensions were fabricated on a commercial SOI wafer with a $340 \mathrm{~nm}$-thick-topsilicon layer and a $2 \mu \mathrm{m}$ BOX layer. The waveguide and SWG cladding are defined with electron beam lithography (EBL) at the same time and subsequently fully etched to the BOX layer with deep reactive ion etching (DRIE) in order to have straight and smooth sidewalls. Then the BOX layer is etched away with buffered oxide etching (BOE).
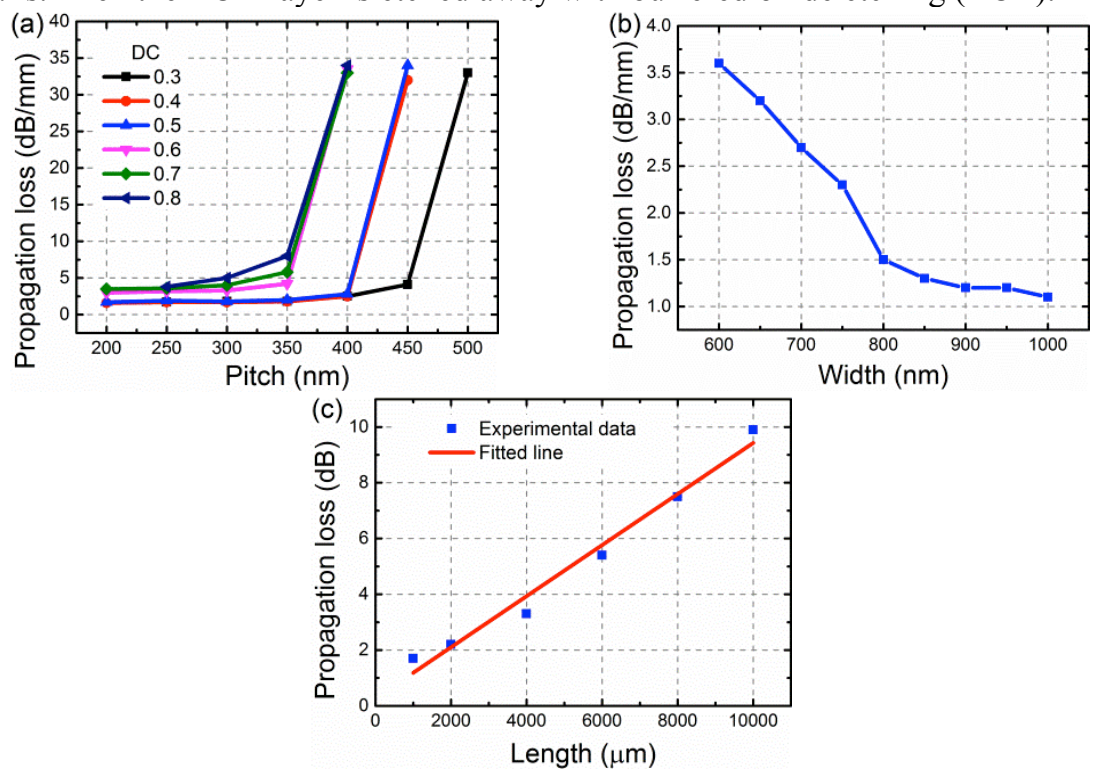

Fig. 2. (a). The propagation loss under different $D C$ and Pitch when $\mathrm{W}_{\mathrm{wg}}=800 \mathrm{~nm}$ and $\mathrm{T}_{\mathrm{si}}=340 \mathrm{~nm}$; (b) The propagation loss under different $\mathrm{W}_{\mathrm{wg}}$ when $\mathrm{DC}=0.3$, Pitch $=350 \mathrm{~nm}$ and $\mathrm{T}_{\mathrm{si}}=340 \mathrm{~nm}$; (c) The experimental measured propagation loss and fitted line when $\mathrm{DC}=0.3$, Pitch $=350 \mathrm{~nm}, \mathrm{~W}_{\mathrm{wg}}=$ $800 \mathrm{~nm}$ and $\mathrm{T}_{\mathrm{si}}=340 \mathrm{~nm}$.

All the characterization is conducted under room temperature with fundamental TE mode. As shown in Fig. 2(a), when $D C=0.3$, Pitch $>450 \mathrm{~nm}$, the propagation loss suddenly increases significantly and reaches the limitation of our detector $(-32 \mathrm{~dB})$ due to the serious diffractive effect and back reflections. With the increase of $D C$ to 0.8 , the cutoff point moves to smaller pitches, which is consistent with the reported simulation results [21]. In order to completely avoid the diffractive effect and back reflections while possess a large exposed area of waveguides and enough mechanical stability, we choose the dimensions as Pitch $=350 \mathrm{~nm}$ and $D C=0.3$. Fig. 2(b) shows the influence of the waveguide width on the propagation loss with Pitch $=350 \mathrm{~nm}$ and $D C=0.3$. As seen, with the increase of width from $600 \mathrm{~nm}$ to $800 \mathrm{~nm}$, the propagation loss drops dramatically. 
But when the width further increases, the decreasing rate of propagation loss becomes slower and then saturated. Although a much wider waveguide $\left(W_{w g}>800 \mathrm{~nm}\right)$ can offer a better confinement and a lower propagation loss, the sensitivity is degraded due to a less energy leakage and in turn a smaller overlap between the evanescent field and the testing chemicals. As a result, we set $W_{w g}=800 \mathrm{~nm}$ in order to have a balance between the propagation loss and the sensitivity. Due to the limitation of experimental setup, the impact of the thickness is not investigated in this work. The shrinking of thickness will put more compression to the mode field from vertical direction, which will result in a larger energy leakage of fundamental TM mode and a higher sensitivity. But this will also result in a higher propagation loss as a larger portion of energy is scattered by the roughness at the upper and lower surfaces. With all the above optimized parameters and $T_{s i}=340 \mathrm{~nm}$, the propagation loss has been plotted and fitted in Fig. 2(c). As seen, the propagation loss of the one-time suspended waveguide is about $9.2 \mathrm{~dB} / \mathrm{cm}$.

Based on the above suspended waveguide design $\left(\right.$ Pitch $=350 \mathrm{~nm}, D C=0.3, W_{w g}=$ $800 \mathrm{~nm}$, and $T_{s i}=340 \mathrm{~nm}$ ), the suspended microracetrack resonator was fabricated and characterized. The scanning electron microscope (SEM) images of the fabricated device have been shown in Fig. 3. The coupling length $(C L)$ and the gap width $\left(W_{g}\right)$ between the bus waveguide and the microracetrack are set as $10 \mu \mathrm{m}$ and $200 \mathrm{~nm}$ respectively. The radius of the bendings ( $R$ ) is set as $50 \mu \mathrm{m}$.

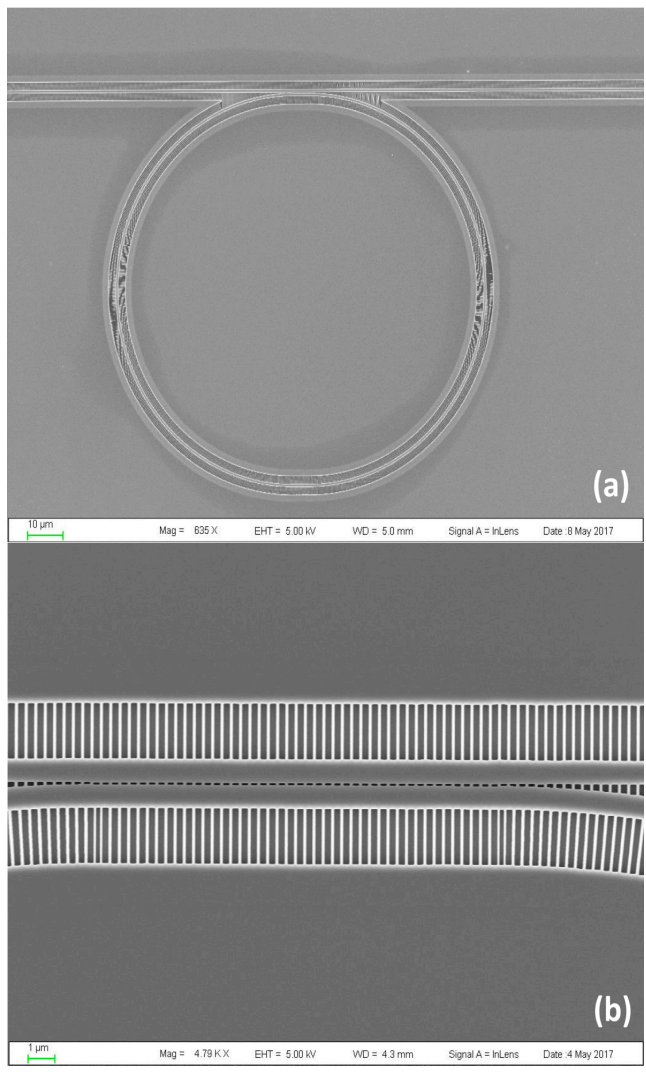

Fig. 3. (a). The overview SEM image of the one-time etching suspended microracetrack resonator; (b) The magnified SEM image of the coupling region. 
The experimental and theoretical fitting results are shown in Fig. 4. The fitting numerical model is specially developed based on the equivalent refractive index method $[14,15,26,27]$. As Pitch $=350 \mathrm{~nm}$ is small enough, the equivalent refractive index of the lateral SWG metamaterial can be written as:

$$
n_{\text {eq }}=n_{\text {cladding }}+D C^{*}\left(n_{\text {si }}-n_{\text {cladding }}\right)
$$

where $n_{\text {cladding }}$ is the refractive index of the cladding layer, such as air or testing chemicals; $n_{s i}$ is the refractive index of silicon. With this equivalent refractive index of the lateral cladding layer, the effective indices of the suspended waveguide at different wavelengths can be calculated with the BeamPROP module of Rsoft software by taking the dispersion into account. Then by substituting the effective indices into our numerical model and tuning the coupling coefficient $k$ and round-trip power attenuation $\alpha^{2}$, the fitted transmission spectrum can be obtained. The fitting parameters are: $k=0.375 \mathrm{i}, \alpha^{2}=0.9116$. From the measurements, the full-width-at-half-maximum (FWHM) is about $0.12 \mathrm{~nm}$, which corresponds to a quality factor ( $\mathrm{Q}$ factor) of 16600 . The extinction ratio (ER) is about 12.3 $\mathrm{dB}$.

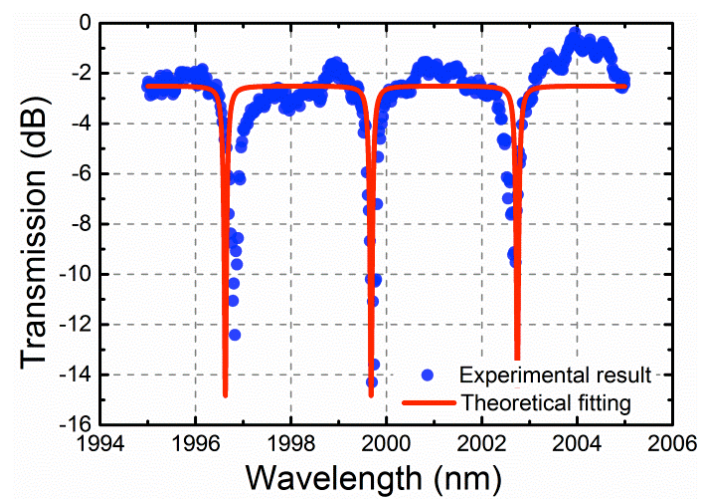

Fig.4. The experimental and theoretical fitting results of the one-time etching suspended microracetrack resonator with SWG metamaterial cladding under $\mathrm{CL}=10 \mu \mathrm{m}, \mathrm{W}_{\mathrm{g}}=200 \mathrm{~nm}$, and $\mathrm{R}=$ $50 \mu \mathrm{m}$.

Here we calculated the sensitivity with the ethanol-water solutions of different concentrations $(0 \% \sim 4 \%)$. The refractive index of pure water is 1.306 at $2 \mu \mathrm{m}$ wavelength. When the concentration of ethanol increases by $1 \%$, the refractive index of the ethanolwater solution increases by 0.00047 . As shown in Fig. 5, by substituting the fitted parameters and the different equivalent refractive indices of the cladding layer into our numerical model, the transmission spectra can be calculated. By measuring the wavelength shifting of the resonant dips, the sensitivities can be obtained as $58 \mathrm{~nm} / \mathrm{RIU}$ and 303 $\mathrm{nm} / \mathrm{RIU}$ for fundamental TE and TM mode, respectively. The moderate sensitivity of fundamental TE mode is due to the lattice structure at the two sides of waveguide, which covers the area at the connection point with the waveguide. And less exposed area of the waveguide results in smaller overlap between the evanescent field and the testing chemicals. If the BOX layer can be etched away with vapor HF to avoid the releasing stress, thinner silicon pillars can be fabricated and more exposed area can be obtained. Besides, by reducing the width of waveguide, the mode field is compressed and more energy will gather at the side walls, which also can improve the sensitivity but increase the propagation loss at the same time. 

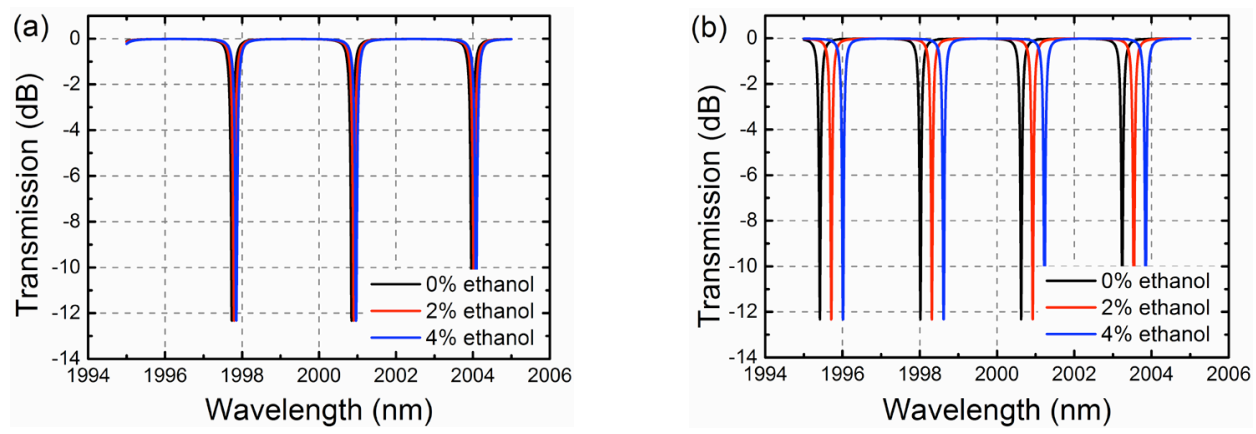

Fig. 5. (a) The normalized transmission spectra of fundamental TE mode under the ethanol-water solutions of different concentrations; (b) The normalized transmission spectra of fundamental TM mode under the ethanol-water solutions of different concentrations

\section{Conclusion}

In this manuscript, for the first time, we have theoretically and experimentally demonstrated a one-time etching suspended microracetrack resonator with lateral SWG metamaterial cladding for MIR bio-chemical sensing applications. With the advantage of suspended structure, the overlap between the evanescent field and the testing chemicals is greatly enhanced, which is helpful for improving the sensitivity. The one-time etching process also makes it easier to fabricate. Various one-time etching suspended waveguides with different dimensions were fabricated and characterized. The propagation loss of the optimized suspended waveguide is experimentally measured as $\sim 9.2 \mathrm{~dB} / \mathrm{cm}$. Based on the optimized suspended waveguide, the one-time etching suspended microracetrack resonator was fabricated and tested. The experimental transmission spectrum is well fitted with our specially developed numerical model. The ER is $\sim 12.3 \mathrm{~dB}$. The FWHM is about $0.12 \mathrm{~nm}$ which corresponds to a $\mathrm{Q}$ factor of $\sim 16600$. With the equivalent refractive index method and our numerical model, the expected sensitivities of fundamental TE and TM mode were calculated as $58 \mathrm{~nm} / \mathrm{RIU}$ and $303 \mathrm{~nm} / \mathrm{RIU}$ respectively. The results presented in this manuscript show a great potential of the one-time etching suspended microracetrack resonator with SWG metamaterial cladding for MIR integrated optical bio-chemical sensing applications.

\section{References}

1. K. D. Vos, I. Bartolozzi, E. Schacht, P. Bienstman, and R. Baets, Opt. Express, 15, 12, 7610-7615, (2007).

2. K. D. Vos, J. Girones, S. Popelka, E. Schacht, R. Baets, and P. Bienstman, Biosensors and Bioelectronics, 24, 8, 2528-2533, (2009).

3. A. L. Washburn, L. C. Gunn, and R. C. Bailey, Anal. Chem., 81, 22, 9499-9506, (2009).

4. A. L. Washburn, M. S. Luchansky, A. L. Bowman, and R. C. Bailey, Anal. Chem., 82, 1, 69-72, (2010).

5. J. G. W.-Pérez, P. Cheben, A. Ortega-Moñux, C. A.-Ramos, D. P.-Galacho, R. Halir, I. M.-Fernández, D.-X. Xu, and J. H. Schmid, Opt. Lett., 39, 15, 4442-4445, (2014).

6. X. Wang, J. Flueckiger, S. Schmidt, S. Grist, S. T. Fard, J. Kirk, M. Doerfler, K. C. Cheung, D. M. Ratner, and L. Chrostowski, J. Biophoton., 6, 10, 821-828, (2013). 
7. S. T. Fard, V. Donzella, S. A. Schmidt, J. Flueckiger, S. M. Grist, P. T. Fard, Y. Wu, R. J. Bojko, E. Kwok, N. A. F. Jaeger, D. M. Ratner, and L. Chrostowski, Opt. Express, 22, 12, 14166-14179, (2014).

8. W. C. Jiang and Q. Lin, Proc. SPIE, 9367, Silicon Photonics X; 936708, (2015).

9. T. Claes, J. G. Molera, K. D. Vos, E. Schacht, R. Baets. and P. Bienstman, IEEE Photonics J., 1, 3, 197-204, (2009).

10. K. B. Gylfason, C. F. Carlborg, A. Kaźmierczak, F. Dortu, H. Sohlström, L. Vivien, C. A. Barrios, W. V. D. Wijngaart, and G. Stemme, Opt. Express, 18, 4, 3226-3237, (2010).

11. P. J. Bock, P. Cheben, J. H. Schmid, J. Lapointe, A. Delâge, S. Janz, G. C. Aers, D.-X. Xu, A. Densmore, and T. J. Hall, Opt. Express, 18, 19, 20251-20262, (2010).

12. J. Wang, I. Glesk, and L. R. Chen, Opt. Express, 22, 13, 15335-15345, (2014).

13. V. Donzella, A. Sherwali, J. Flueckiger, S. T. Fard, S. M. Grist, and L. Chrostowski, Opt. Express, 22, 17, 21037-21050, (2014).

14. V. Donzella, A. Sherwali, J. Flueckiger, S. M. Grist, S. T. Fard, and L. Chrostowski, Opt. Express, 23, 4, 4791-4803, (2015).

15. J. Flueckiger, S. Schmidt, V. Donzella, A. Sherwali, D. M. Ratner, L. Chrostowski, and K. C. Cheung, Opt. Express, 24, 14, 15672-15686, (2016).

16. K. Miyamoto, K. Ishibashi, K. Hiroi, Y. Kimura, H. Ishii, and M. Niwano, Appl. Phys. Lett., 86, 053902, (2005).

17. F. K. Tittel, Y. Bakhirkin, A. Kosterev, R. Lewicki, S. So, G. Wysocki, and R. F. Curl, Proc. SPIE, 6900, 69000Z, (2008).

18. M. Sieger and B. Mizaikoff, Anal. Chem., 88, 11, 5562-5573, (2016).

19. R. Soref, Nat. Photonics, 4, 495-497, (2010).

20. Z. Cheng, X. Chen, C. Y. Wong, K. Xu, C. K. Y. Fung, Y. M. Chen, and H. K. Tsang, Opt. Lett., 37, 7, 1217-1219, (2012).

21. J. S. Penadés, C. A.-Ramos, A. Z. Khokhar, M. Nedeljkovic, L. A. Boodhoo, A. O.Moñux, I. M.-Fernández, P. Cheben, and G. Z. Mashanovich, Opt. Lett., 39, 19, 56615664, (2014).

22. J. S. Penades, A. O.-Moñux, M. Nedeljkovic, J. G. W.-Pérez, R. Halir, A. Z. Khokhar, C. A.-Ramos, Z. Qu, I. M.-Fernández, P. Cheben, and G. Z. Mashanovich, Opt. Express, 24, 20, 22908-22916, (2016).

23. Z. Cheng, X. Chen, C. Y. Wong, K. Xu, and H. K. Tsang, IEEE Photon. J., 4, 5, 1510$1519,(2012)$.

24. Y. Xia, C. Qiu, X. Zhang, W. Gao, J. Shu, and Q. Xu, Opt. Lett., 38, 7, 1122-1124, (2013). 